\title{
PARAMETERS OPTIMIZATION FOR CREATING RELIABLE PHOTOGRAMMETRIC MODELS IN EMERGENCY SCENARIOS
}

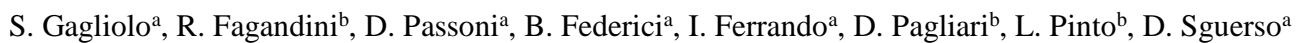 \\ a Università degli Studi di Genova, DICCA - Laboratory of Geodesy, Geomatics and GIS, Via Montallegro 1, 16145 Genoa \\ (sara.gagliolo, ilaria.ferrando)@edu.unige.it, daniele.passoni@dicca.unige.it, (bianca.federici, domenico.sguerso)@unige.it

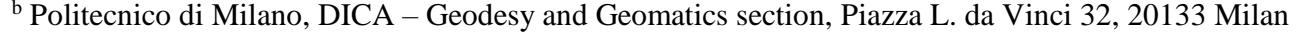 \\ (roberta.fagandini, diana.pagliari, livio.pinto)@polimi.it
}

KEY WORDS: UAS, Photogrammetry, Emergency, Survey strategy, Cultural Heritage, Safety, Optimization

\begin{abstract}
:
An optimized planning and realization of the survey, coupled with well thought-out processing, allows obtaining good quality results, while guaranteeing a reasonable use of resources and time. It represents a benefit for both operators and end-users. The former can save time and acquire smaller datasets to process, while the latter can invest their resources better. These goals are even more important in case of an emergency, because the circumstances can quickly change, causing risk to both people and goods. The paper examines the possibility of using Unmanned Aerial Systems (UAS) photogrammetry for 3D modelling in such scenario, focusing on finding a compromise between the final accuracy and the requested processing time. An experimental test has been conducted over the Castle of Casalbagliano, a damaged structure located near Alessandria (Piedmont, Italy), simulating a postemergency scenario. Several processing strategies have been tested to define a workflow useful in this kind of situations. The quality of the different processing has been evaluated in terms of both residuals of the bundle block adjustment and quality of the generated dense point cloud, compared with a reference Terrestrial Laser Scanner acquisition. Finally, the possibility of publishing the obtained $3 \mathrm{D}$ models on the web has been exploited too.
\end{abstract}

\section{INTRODUCTION}

In case of a catastrophic event damaging an area, several aspects must be taken into account. The first and main goal is to save human lives, by taking urgent actions to help people move away from danger. The second important task is the preservation of goods. The present research has been developed with a focus on the latter aspect, paying particular attention to the preservation of cultural and artistic heritage, especially buildings.

The interest of researchers on cultural heritage has widespread in the last decades, focusing on different aspects: from pure historical image documentation (Yastikli, 2007; Yilmaz, 2007), mapping (Remondino, 2011) and digital reconstruction of destroyed objects (Grün et al., 2004) to the creation of web-catalogues and virtual museum tours (Wojciechowski, 2004; Bruno et al., 2010).

3D models of cultural heritage could help in securing, planning and performing the restoration of damaged buildings. They are commonly produced by Terrestrial Laser Scanner (TLS) surveys, Unmanned Aerial Systems (UAS) or terrestrial photogrammetry.

Among all the different survey techniques UAS photogrammetry can guarantee the production of 3D models at a high level of precision and accuracy, required to ensure building conservation. At the same time, they can facilitate the access in areas where otherwise it would be impossible to enter. Furthermore, the used instrumentation is cheap and the survey operations are quick. Hence, its use is very common and there is a plenty of applications in different fields (e.g. for architectural applications refer to: Themistocleous, 2015; Sauerbier and Eisenbeiss, 2010; Brutto et al., 2014).

In recent years, the task of emergency management has become very crucial in Italy, as it has hit by many earthquakes, with serious damages to goods and significant losses of human lives.

Several authors have considered as case studies structures damaged by seismic events, giving a substantial contribution in the securing of sites and in the restoration of buildings by means of effective and accurate metrical surveys. Some recent examples are here mentioned, grouped by the employed survey techniques: Chiabrando et al. (2017) and Gagliolo et al., (2017) performed UAS photogrammetry to survey artistic buildings damaged by Centre Italy earthquakes (August - October 2016); Ballarin et al., (2017) used TLS to build a 3D model of the Castle of San Giorgio in Mantua, hit by Emilia Romagna earthquake (May 2012); integrated techniques (traditional survey, laser scanning and terrestrial or UAS photogrammetry) were applied by Oreni et al., (2017), Fregonese et al. (2017), Achille et al. (2015) and Ballarin et al. (2013) after the L'Aquila earthquake (April 2009) and the Emilia Romagna earthquake (May 2012).

Concerning case studies outside Italy, faced with the help of integrated techniques, $\mathrm{Xu}$ et al. (2014) and Meyer et al. (2015) regard Lushan Earthquake (2013, Sichuan, China) and South Napa Earthquake (2014, California, USA), respectively.

Other researches focused on the analysis of sites affected by earthquakes prior to those already mentioned, in order to assess the evolution of damages and the suitable strategies for enhancement (Radicioni et al., 2017; Barone et al., 2017).

The main goal of the work discussed in the present paper is to create a step-by-step operative workflow for generating different photogrammetric products (namely dense point clouds and orthophotos) quickly and easily.

In addition, the possibility of Web publication of the photogrammetric products has been exploited, ensuring their worldwide distribution and easy access. The investigated 
web-based tool could enable the end-users to carry out quick queries and simple analysis of 3D models (e.g. section extraction or distance measurements), while highlighting the metrical accuracy of the generated products. Moreover, it could support the local authority and/or Civil Protection to protect the sites quickly, using fewer resources and making the operations more effective.

To simulate an emergency scenario and to face the real difficulties imposed by such context, the ruins of a castle were chosen as case study. Of course, in case of real applications, it is mandatory to take into account the specific conditions of the surveyed area and carry on an on-site deep analysis.

The paper is organized as follows: in Section 2 the selected test area, the planning and the realization of the photogrammetric flights are presented. Few hints about the photogrammetric data processing in different scenarios and the estimated precision are briefly described (for more details, refer to Gagliolo et al., 2017). Section 3 focuses on dense cloud generation and on the requested computational time. In Section 4, the procedure to share the created models on a Web platform is shown. Final considerations and future proposals are discussed in Section 5.

\section{PREVIOUS EXPERIENCES IN THE TEST AREA}

The case study is the Castle of Casalbagliano (Fig. 1), located in the countryside of Alessandria (Piedmont, Italy). The castle was built at the end of XIII century by the Bagliani's lineage. The toponym has been recently attributed in memory of the founder family.

The investigated area has been object of a preliminary inspection, to carefully plan data acquisition. The castle has a square map with the side of about $30 \mathrm{~m}$, inaccessible inside. Then, the survey campaign was carried out in one single day.

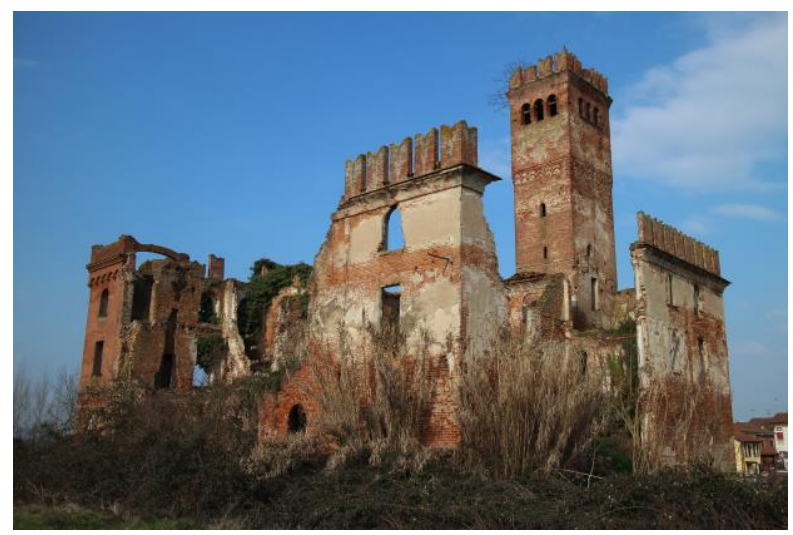

Fig. 1 Castle of Casalbagliano

\subsection{Survey campaign}

The survey has been performed testing different acquisition techniques and comparing the final metric accuracy.

Total Station (TS) (Leica Nova MS60 Multistation) and Global Navigation Satellite System (GNSS) receiver (Topcon Hiper Pro) were used to measure the coordinates of the Ground Control Points (GCPs), while UAS Photogrammetry and TLS (Z+F Imager ${ }^{\circledR}$ 5006h) were used to survey the object.

Concerning the photogrammetric survey, the images have been acquired using a Canon EOS-M camera, with a fixed focal length of $22 \mathrm{~mm}$. Three different data sets have been collected.

In the first and second datasets the camera has been installed on a Microcopter hexacopter, equipped with an on board low-cost GNSS receiver (Ublox LEA GH), a triaxial magnetometer and a Micro Electro-Mechanical System/Inertial Measurement Unit (MEMS/IMU). The UAS maximum payload is about $0.5 \mathrm{~kg}$ and it has a flight autonomy of about $15 \mathrm{~min}$.

The acquisition of the first dataset was nadiral, at an average flight height of about $40 \mathrm{~m}$. The flight has been planned to guarantee a high overlapping ( $80 \%$ along flight direction and $70 \%$ along cross direction), resulting in an average Ground Sample Distance (GSD) of about $9 \mathrm{~mm}$.

The second flight was realized using an oblique camera configuration (tilt angle equal to $45^{\circ}$ ), describing a square around the castle. The flight height has been set equal to 50 $\mathrm{m}$, guaranteeing an overlapping of about $80 \%$.

The third dataset has been acquired using a terrestrial photogrammetry configuration.

The photogrammetric block has been georeferenced using 19 GCPs, materialized by black and white square targets $(0.3 \mathrm{~m}$ side) and divided into three different groups (see Fig. 2): 15 GCPs used in the first test case (indicated in red and green), 12 GCPs used in the second test case (depicted in green), and 3 GCPs used in the third test case (represented by stars). Four points have been used as Check Points (CPs) (blue squares), to evaluate the quality of the bundle block adjustment.

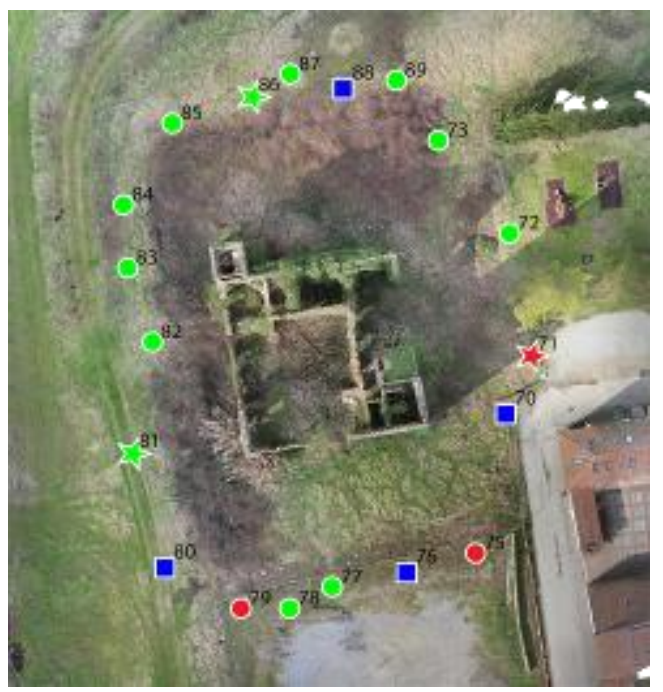

Fig. 2 Locations of the GCPs and CPs over the surveyed area. The blue squares represent the 4 CPs. The 15 GCPs of first test case are reported in red and green, the 12 GCPs of the second test case are depicted in green and the 3 GCPs of the third test case are represented by stars

Eight station points have been located around the castle, as shown in Fig. 3, both to perform a TLS survey of the ruins and for measuring the coordinates to the GCPs by TS collimations. 


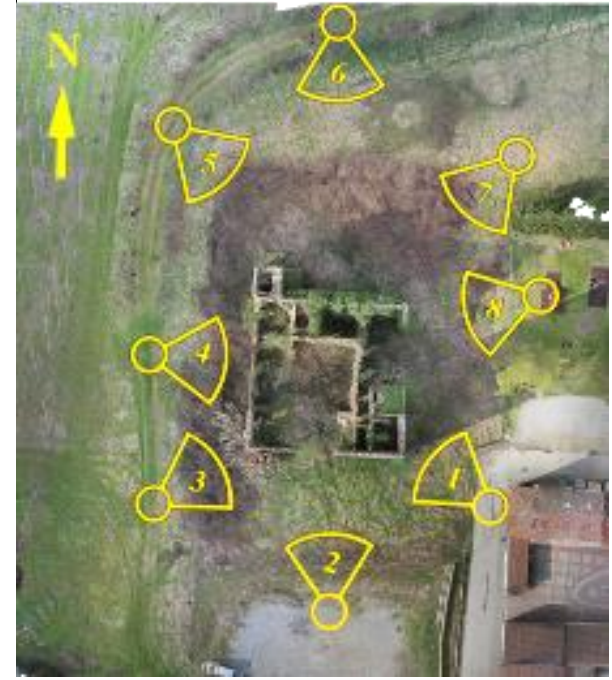

Fig. 3 Locations of station points used for the laser scanner acquisition and for the determination of the geodetic network

The TS is characterized by an angular accuracy of $3^{\mathrm{cc}}$ and by a distance accuracy of $1 \mathrm{~mm}+1.5 \mathrm{ppm}$ (using reflective prism), when it is used in TS mode (Fagandini et al., 2017).

The local reference system has been defined with its origin in the point 1 , the $y$-axis oriented towards point 8 (i.e. approximately parallel to one of the perimeter walls), the $\mathrm{z}$ axis oriented along the vertical and the $\mathrm{x}$-axis to complete the Cartesian orthogonal right-handed triad. The geodetic network has been adjusted using the commercial Infinity software, supplied by the TS manufacturer, resulting in a closing angle error of $50^{\mathrm{cc}}$

The target coordinates have been measured also by GNSS positioning in NRTK mode, reaching an accuracy of $0.02 \mathrm{~m}$.

The TLS scans have been performed at a resolution of 20,000 points $/ 360^{\circ}$, providing a spacing of about $1 \mathrm{~cm}$ in the acquired point clouds. In order to align the different scans, 32 Forex reflective black and white targets have been placed in the surveyed area. This was necessary despite the presence of photogrammetric GCPs, due to the impossibility of seeing them clearly in the TLS scans.

The targets have been placed on different objects located around the scene (e.g. perimeter fences, trees etc.), distributed in all directions, ensuring better results in the scans alignment phase. The target coordinates have been measured with the TS, maintaining the highest possible redundancy, and adjusted in the geodetic network.

Finally, additional scans have been performed using the TS in scanning mode, stationing on the same points used for the realisation of the geodetic network.

More details on survey planning and data acquisition are given in Gagliolo et al. (2017).

\subsection{Data processing}

All the acquired photogrammetric blocks have been processed with the commercial software Agisoft PhotoScan $($ ) - version 1.2.6 (http://www.agisoft.com/), using the standard workflow proposed by the software itself. All the analyses discussed in the present paper have been carried out using a desktop Personal Computer (64 bits Windows 7 operating system, Intel ${ }^{\circledR}$ core $^{\mathrm{TM}} \mathrm{i} 7-4770 \mathrm{CPU} @ 340 \mathrm{GHz}$ processor and 32 GB of RAM, Intel® HD Graphics 4600, 20 Cores @ 400 $\mathrm{MHz}, 1297 \mathrm{MB}$ ). After the solution of the bundle-block adjustment and the estimation of External Orientation (EO) parameters, the self-calibration has been performed through the Optimize Cameras option (for all the considered scenarios). In fact, it is quite important to refine the Internal Orientation (IO) parameters in the case of UAS flights, because they can easily be subject to variations due to the impacts suffered by the lens during take-off and landing.

Different block configurations and GCPs numerousness for image processing have been considered, evaluating the results in terms of final accuracy and computational cost.

The acquired images have been divided into three groups, relying on the specific acquisition geometry: nadir images ( 83 frames; $\mathrm{N}$ ), oblique images (61 frames; $\mathrm{O}$ ) and terrestrial acquisition (28 frames; T). It is worth noting that it is possible to fully reconstruct the whole structure only using the $\mathrm{O}$ dataset. Indeed, the $\mathrm{N}$ and $\mathrm{T}$ images do not give a complete view of the object, because they focus the attention on a specific portion of the building (roofs and façades, respectively). Therefore, it is not possible to realize a complete model using only $\mathrm{N}$ and $\mathrm{T}$ datasets.

Several processing settings have been tested by combining the three image datasets. It is important to point out that the addition of images, taken from $\mathrm{N}$ or $\mathrm{T}$ dataset, to the $\mathrm{O}$ one does not lead to any specific improvement, mainly due to the auto-consistency of the oblique geometry that guarantees a high overlapping and completeness of the final model, thanks to a rigorous planning.

A second variable taken into account was the number and the distribution of GCPs, focusing on three different configurations. In the first case, the whole set of 15 GCPs was used; in the second scenario, 12 GCPs were selected from the previous one, discarding the worst points; finally, the third scenario involved the minimum number of requested GCPs (3). Certainly, the last configuration is the closest to the emergency situations, in which the survey area is not easily accessible.

For all the considered scenarios, the same dataset of CPs was used to evaluate the quality of the photogrammetric solution (see blue squares in Fig. 2). The CPs locations have been set so to obtain a quite regular distribution around the investigated structure. It is worth noting that the structure is not accessible, thus the targets could not be located in the internal area.

The impact of the image resolution on EO parameters estimation has been evaluated too. Two different scenarios were analysed: the use of images at their full resolution $(\mathrm{H})$ and their downscaling by a factor 4 (i.e. 2 times for each side; $\mathrm{M})$, respectively.

The overall quality of the bundle block adjustment obtained in Gagliolo et al. (2017) resulted in about $3 \mathrm{~cm}$ for all the considered scenarios.

\section{OPERATIVE STRATEGY}

The main goal of the work discussed in the present paper is to present a step-by-step operative workflow for generating different photogrammetric products (namely dense point clouds and orthophotos) as quickly as possible, respecting the required precisions. The prospected scenario is an emergency one, which requires rapid response, the use of limited resources and the guarantee of operator safety.

\subsection{Objective definition}

The photogrammetric survey should allow the following actions: (1) detection of the building in its context; (2) acquisition of the volume of the entire object and eventually 
(3) of the ruins to remove or restore them; (4) inspection of historical-artistic-architectural presences for their preservation. These requirements need different levels of depth, resolutions and densities of point clouds.

The influence of the image resolution has been considered, for each block geometry and GCPs numerousness, by using different alignment quality values of the PhotoScan $\odot$ software.

The quality of the computed solution has been evaluated through the Root Mean Square Errors (RMSE) of the CPs. The RMSE are below $3 \mathrm{~cm}$ for all the considered acquisition geometries and GCPs configurations (see Gagliolo et al., 2017).

The 3 GCPs case was taken into account for simulating a critical scenario due to the presence of ruins. Nevertheless, even in this case, the results show that the influence of the photogrammetric block geometry on the quality was negligible if compared to the accuracy requested by an emergency survey, reasonably set to sub-centimetre order of magnitude. This is probably due to the consistency of the $\mathrm{O}$ block geometry, thanks also to the high overlapping of the images.

\subsection{Time processing analysis}

The requested time for processing a dataset is strictly connected to the number of frames that compose the photogrammetric block, the image quality and the availability of image geolocation (position and attitude), useful for image pair pre-selection (the GPS image geotagging was not available in the analysed scenario). The influence of image quality on the processing time (for both EO and dense cloud generation) is here addressed, focusing on the impact of block geometry and GCPs numerousness.

It is worth noting that the absolute requested time for image processing is strictly related also to the used hardware. As PhotoScan ${ }^{\odot}$ is not an open source software, and thus the embedded algorithms are unknown, only general information about the impact of the different parameters can be deduced. Focusing on the requested time for the EO processing, it increases of $30 \%$ considering $\mathrm{H}$ instead of $\mathrm{M}$ image quality. This could be tolerable because the computational time for EO using $\mathrm{H}$ image quality does not exceed one hour. Therefore, the alignment quality has to be chosen considering both the complexity of the object and its texture. In facts, the increase of the quality level corresponds to the research of a higher number of tie points, which does not necessarily guarantee an optimal matching and, consequently, an optimal EO.

Concerning the dense cloud reconstruction, if quick information is necessary for safety operations, a medium image quality is suggested for immediate processing of the dataset on-site. On the other hand, a high image quality is recommended when it is possible to process data in office. In this second case, a maximum processing time of half a day should be considered.

The interpretation of the quality parameters in the dense cloud reconstruction is the same of the accuracy settings given for the EO computation, then $\mathrm{H}$ and $\mathrm{M}$ result in a higher or lower downscaling factor applied on the dataset. In particular, differently from the EO case, high quality corresponds to a downscaling factor of 4 ( 2 times by each side), while the medium one is 16 .

reports the different block geometries for which the dense clouds have been computed, the number of GCPs, together with the time requested to produce the results and the number of generated points. The parameter of time refers only to the dense cloud generation phase. An additional time, lower than an hour in every case, has to be taken into account to consider also the $\mathrm{EO}$ computation.

Table 1 Processing times and number of points for each tested scenario

\begin{tabular}{|c|c|c|c|c|c|}
\hline $\begin{array}{c}\text { Block } \\
\text { geom. }\end{array}$ & $\begin{array}{c}\text { Nr. } \\
\text { GCP }\end{array}$ & $\begin{array}{c}\text { Quality } \\
\text { (EO) }\end{array}$ & $\begin{array}{c}\text { Quality } \\
\text { (Dense } \\
\text { Cloud) }\end{array}$ & $\begin{array}{c}\text { Time } \\
\text { (h:m) }\end{array}$ & $\begin{array}{c}\text { Nr. of } \\
\text { points } \\
\left(\mathbf{1 0}^{6}\right)\end{array}$ \\
\hline $\mathrm{N}+\mathrm{O}+\mathrm{T}$ & 15 & $\mathrm{H}$ & $\mathrm{H}$ & $29: 58$ & 41.7 \\
\hline $\mathrm{N}+\mathrm{O}$ & 15 & $\mathrm{H}$ & $\mathrm{H}$ & $30: 14$ & 42.6 \\
\hline $\mathrm{N}+\mathrm{O}$ & 15 & $\mathrm{H}$ & $\mathrm{M}$ & $4: 15$ & 15.1 \\
\hline $\mathrm{O}$ & 15 & $\mathrm{M}$ & $\mathrm{H}$ & $8: 15$ & 28.3 \\
\hline $\mathrm{O}$ & 15 & $\mathrm{M}$ & $\mathrm{M}$ & $1: 11$ & 7.4 \\
\hline $\mathrm{O}$ & 15 & $\mathrm{H}$ & $\mathrm{H}$ & $11: 07$ & 28.4 \\
\hline $\mathrm{O}$ & 15 & $\mathrm{H}$ & $\mathrm{M}$ & $1: 30$ & 7.7 \\
\hline $\mathrm{O}$ & 12 & $\mathrm{M}$ & $\mathrm{H}$ & $8: 21$ & 30.2 \\
\hline $\mathrm{O}$ & 12 & $\mathrm{M}$ & $\mathrm{M}$ & $1: 09$ & 7.9 \\
\hline $\mathrm{O}$ & 12 & $\mathrm{H}$ & $\mathrm{H}$ & $11: 28$ & 29.1 \\
\hline $\mathrm{O}$ & 12 & $\mathrm{H}$ & $\mathrm{M}$ & $1: 35$ & 7.5 \\
\hline $\mathrm{O}$ & 3 & $\mathrm{M}$ & $\mathrm{H}$ & $8: 31$ & 29.1 \\
\hline $\mathrm{O}$ & 3 & $\mathrm{M}$ & $\mathrm{M}$ & $1: 10$ & 7.6 \\
\hline $\mathrm{O}$ & 3 & $\mathrm{H}$ & $\mathrm{H}$ & $11: 19$ & 28.0 \\
\hline $\mathrm{O}$ & 3 & $\mathrm{H}$ & $\mathrm{M}$ & $1: 34$ & 7.8 \\
\hline
\end{tabular}

Obviously, the requested time for processing $\mathrm{N}+\mathrm{O}+\mathrm{T}$ and $\mathrm{N}+\mathrm{O}$ is higher, with respect to $\mathrm{O}$ case. Furthermore, as already mentioned, the $\mathrm{O}$ case was already auto-consistent hence the contribution of added images cannot be appreciated. However, the number of generated points is higher for $\mathrm{N}+\mathrm{O}+\mathrm{T}$ and $\mathrm{N}+\mathrm{O}$, because of the presence of multiple points of view.

The analysis of the $\mathrm{O}$ case shows that the image quality selected for the EO phase influences the requested time for the dense cloud generation. Comparing the solutions obtained using the same dense cloud quality but different EO image resolutions ( $\mathrm{M}$ vs $\mathrm{H}$ 'alignment'), the computational time increases of about $20 \%$, which can be considered tolerable to obtain higher precision. However, the number of points composing the dense clouds does not change. Furthermore, no impact connected to the number of GCPs has been registered.

The dimension of the point clouds generated by $\mathrm{H}$ image quality is about four times higher than the ones generated by $M$ quality, in accordance to the ratio of the downscaling factors applied to the images. Nevertheless, the latter requires $1 / 7$ of the computational time of the former. These differences are substantial in emergency scenarios, affecting the choice of the processing parameters. In particular, it seems preferable to use a medium image quality for the generation of the dense cloud. The high image quality setting requires a computational time which results excessive to obtain an immediate solution on site, but it is not optimal also to perform a fast processing in office.

Considering $\mathrm{M}$ image quality for both $\mathrm{EO}$ and dense cloud computation, the total computational time is about 2 hours, which is often still too high for the needs of on-site processing.

For all these reasons, another combination has been tested, setting low image quality (L) for the dense cloud generation (downscaling factor of 64). The results obtained using 15 GCPs, $\mathrm{H}$ image quality for EO, oblique dataset and varying the dense cloud quality are summarized in . 
Table 2 Processing time and number of points for the combinations with only $\mathrm{O}$ images, 15 GCPs, high image quality for EO and different image quality to produce dense cloud

\begin{tabular}{|c|c|c|c|c|c|}
\hline $\begin{array}{c}\text { Block } \\
\text { geom. }\end{array}$ & $\begin{array}{c}\text { Nr. } \\
\text { GCP }\end{array}$ & $\begin{array}{c}\text { Quality } \\
\text { (EO) }\end{array}$ & $\begin{array}{c}\text { Quality } \\
\text { (Dense } \\
\text { Cloud) }\end{array}$ & $\begin{array}{c}\text { Time } \\
\text { (h:m) }\end{array}$ & $\begin{array}{c}\text { Nr. of } \\
\text { points } \\
\left(\mathbf{1 0}^{6}\right)\end{array}$ \\
\hline $\mathrm{O}$ & 15 & $\mathrm{H}$ & $\mathrm{H}$ & $11: 07$ & 28.4 \\
\hline $\mathrm{O}$ & 15 & $\mathrm{H}$ & $\mathrm{M}$ & $1: 30$ & 7.7 \\
\hline $\mathrm{O}$ & 15 & $\mathrm{H}$ & $\mathrm{L}$ & $0: 15$ & 1.9 \\
\hline
\end{tabular}

The $\mathrm{L}$ solution requires a computational time of about $20 \%$ of that for $\mathrm{M}$ and about $2 \%$ of that for $\mathrm{H}$ image quality. In this case, the appropriate speed for the on-site operations is clearly guaranteed, but resulting in a detriment of the resolution of the generated point cloud.

Of course, a unique level of detail cannot be sufficient to meet all the stakeholders' requirements, especially if highresolution results are needed. In order to pursue the optimal processing strategy, it is important to answer to two main questions: (1) What are the variations in point clouds density between $\mathrm{M}$ and $\mathrm{H}$ image quality? (2) What is the proper image quality to achieve the different resolution scales needed to describe the object, its architectonical details and its relationship with the context?

Therefore, the proposal of the authors is to diversify the processing, focusing on the satisfaction of each stakeholder, thus identifying the most appropriate quality of processing. In this way, it is possible to avoid worthless processing (e.g. excessive high quality), which saves time. Of course, such diversification requires a substantial knowledge of the photogrammetric problem and of the software characteristics by the operator, whose work could not be limited to the launch of the different phases that compose a standard processing workflow. In section 3.3 the evaluation of the quality of dense clouds in terms of their density is analysed. This task could be very useful for defining the best strategy to follow, according to the desired levels of detail.

\subsection{Point clouds density}

The density of the dense point clouds, generated using the configuration with 3 GCPs and the H EO quality, has been evaluated considering the number of points in about a square meter. Six samples were taken into account in different areas of the Castle façades, at different heights as shown in Fig. 4Fig. 7.

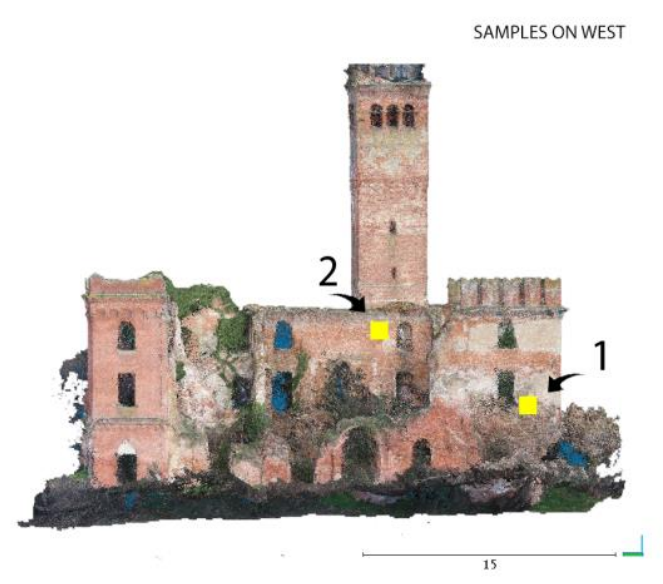

Fig. 4 Samples on West façade

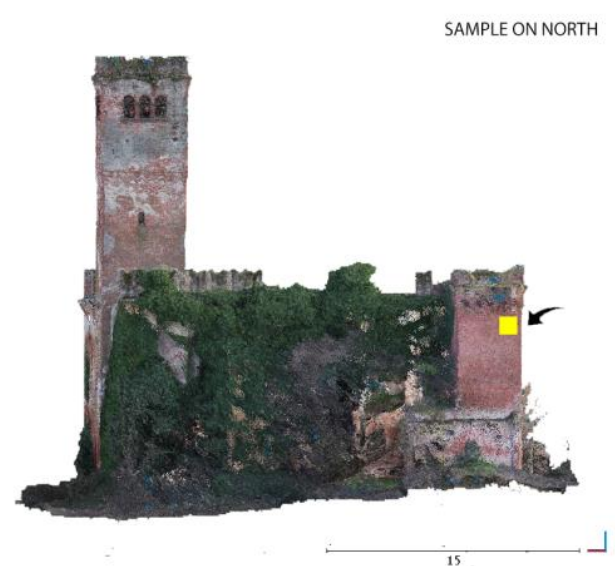

Fig. 5 Sample on North façade

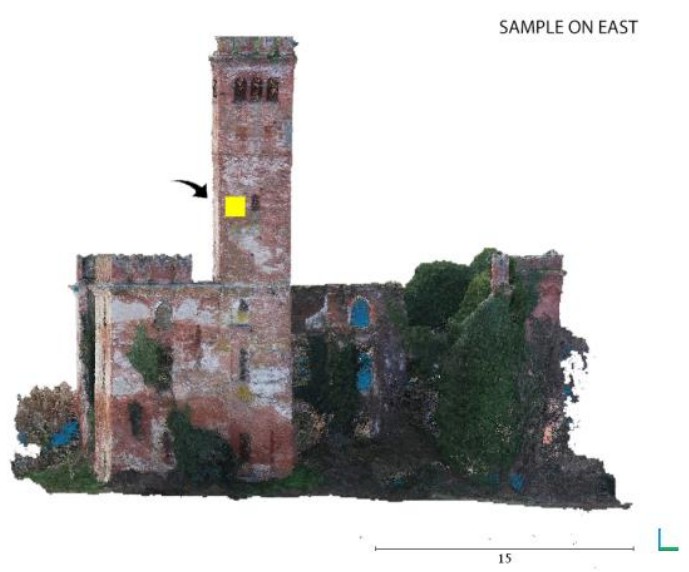

Fig. 6 Sample on East façade

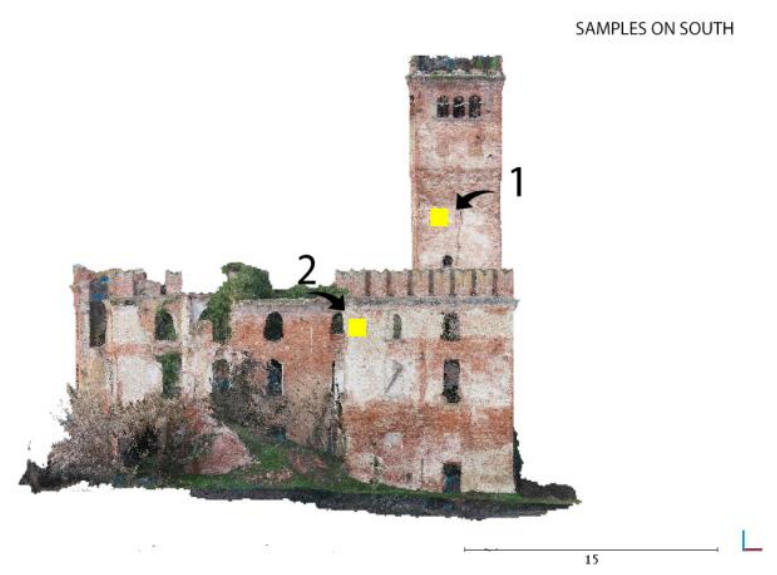

Fig. 7 Samples on South façade

In

, the number of points per square meter and the space between them are reported for each sample. Note that there are no significant differences among the considered samples.

Table 3 Density of the considered samples on the Castle's façades (L, $\mathrm{M}$ and $\mathrm{H}$ indicate the image quality) 


\begin{tabular}{|c|c|c|c|c|c|c|}
\hline & \multicolumn{3}{|c|}{${\text { Points per } \mathbf{~}^{2}}^{2}$} & \multicolumn{3}{c|}{$\begin{array}{c}\text { Linear space between } \\
\text { points (cm) }\end{array}$} \\
\hline $\begin{array}{c}\text { Sample } \\
\text { origin }\end{array}$ & L & M & H & L & M & H \\
\hline W 1 & 144 & 529 & 2057 & 8.3 & 4.3 & 2.2 \\
\hline W 2 & 132 & 552 & 2127 & 8.7 & 4.3 & 2.2 \\
\hline N & 128 & 474 & 1343 & 8.8 & 4.6 & 2.7 \\
\hline E & 145 & 554 & 2148 & 8.3 & 4.2 & 2.2 \\
\hline S 1 & 128 & 591 & 2292 & 8.8 & 4.1 & 2.1 \\
\hline S 2 & 160 & 836 & 3233 & 7.9 & 3.5 & 1.8 \\
\hline
\end{tabular}

Considering a GSD of $9 \mathrm{~mm}$, the spacing between points is about 9-10 times the GSD for L, about 4-5 times for M, and 2-3 times for $\mathrm{H}$.

A dense cloud at UltraHigh image quality (UH) was calculated only for the tower, because of the long computational time and the high computational effort of the whole processing. For the sample on the East façade, 20,920 points per $\mathrm{m}^{2}$ were generated, with a linear spacing between points of $0.7 \mathrm{~cm}$. Hence, the UH quality could only be useful if the aim is to obtain a high detail of a very limited portion of the investigated object, by creating a reduced bounding box.

\subsection{Procedure validation}

The generated photogrammetric dense clouds have been validated by comparing them with a TLS scan, used as reference. The comparison has been performed for the $\mathrm{O}$ case, considering 3 GCPs and H EO quality. This configuration has been analysed because it represents the minimum number of points needed to obtain a 3D fit between the two compared clouds. It is mandatory to underline that the validation procedure is independent from the Reference Frame (RF).

The TLS scans have been pre-processed, oriented and exported using the software supplied by the TLS manufacturer. Then, they have been aligned using at least 8 targets and refined using the ICP (Iterative Closest Point) algorithm. The TLS point clouds have been manually edited in CloudCompare (www.cloudcompare.org) to remove outliers and noise due to the vegetation. The coordinates of the TLS targets have been acquired with the TS, resulting in final point clouds directly co-registered in the same RF of the UAS Photogrammetry survey.

The correspondence between each photogrammetric dense cloud (namely L, M and $\mathrm{H}$ dense cloud quality) and the TLS scan has been computed considering the shortest Euclidean distance (M3C2 algorithm; Lague et al., 2013). The points located at distances greater than the 95th percentile have been excluded. In fact, they have been considered outliers mainly due to the presence of vegetation.

In case of the low quality dense cloud, a subsample of the TLS cloud has been defined before the comparison, in order to obtain a comparable space between points (about $9 \mathrm{~cm}$ ).

In the following Fig. 8Fig. 10, the Gaussian distribution of the differences are represented; the range is limited within $\pm 10 \mathrm{~cm}$, where the largest number of points is located. These diagrams show a good global behaviour of the clouds, in facts the values are typically included between $-3 \mathrm{~cm}$ and +3 $\mathrm{cm}$ for all the configurations, with a lower standard deviation for the high quality.

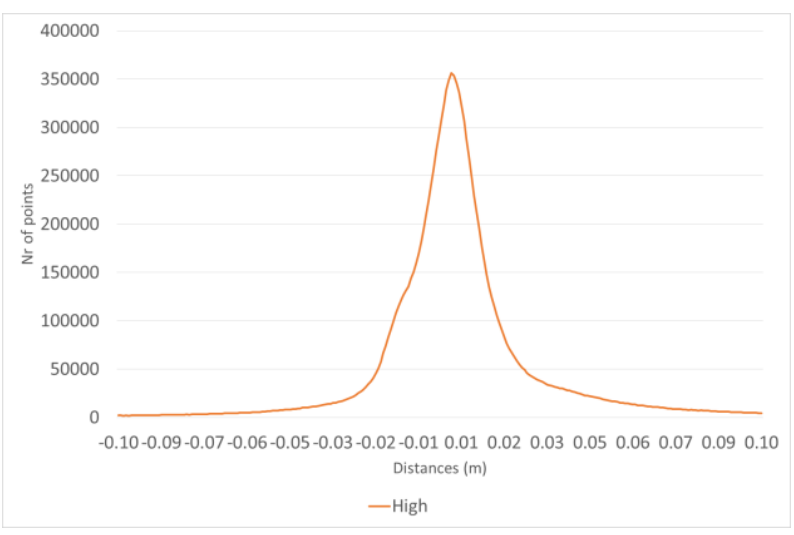

Fig. 8 Distribution of distances $(m)-H$ dense cloud quality; mean $=0.5 \mathrm{~cm}, \mathrm{std}=2.3 \mathrm{~cm}$

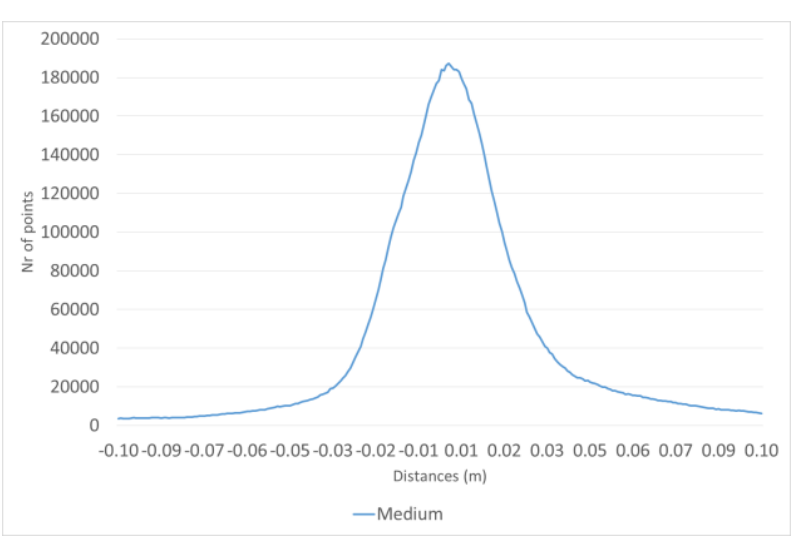

Fig. 9 Distribution of distances (m) - M dense cloud quality; mean $=0.6 \mathrm{~cm}, \mathrm{std}=2.8 \mathrm{~cm}$

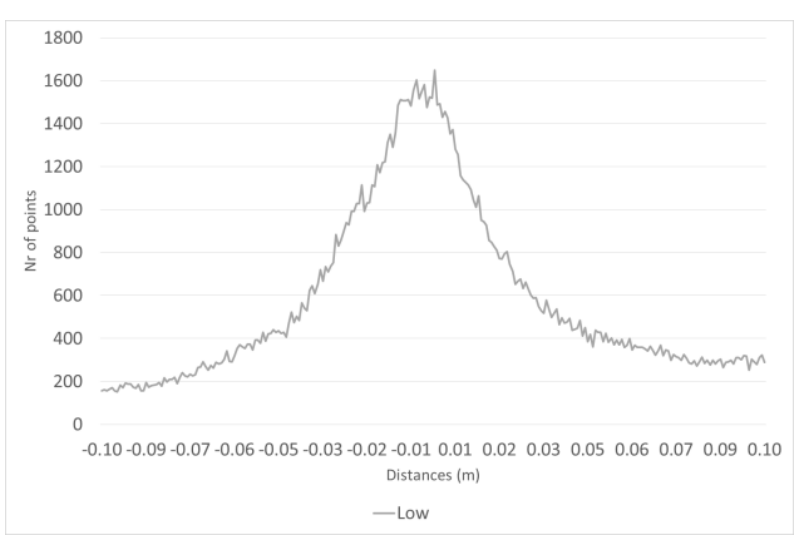

Fig. 10 Distribution of distances $(\mathrm{m})-\mathrm{L}$ dense cloud quality; mean $=0.1 \mathrm{~cm}, \mathrm{std}=4.2 \mathrm{~cm}$

In Fig. 121Errore. L'origine riferimento non è stata trovata. 3 , the UAS photogrammetric point clouds realized with different image quality (L, M and $\mathrm{H}$ respectively) are shown. The colour scale represents the Euclidean distance with respect to the reference TLS scan, expressed in centimetres, set within the range of $\pm 20 \mathrm{~cm}$, to highlight the location of the highest differences in the $\mathrm{M}$ and $\mathrm{H}$ cases. 


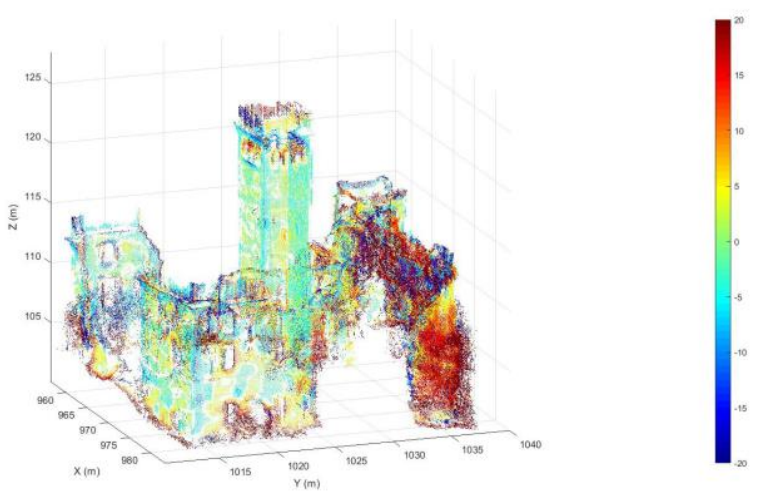

Fig. 11 Differences (in centimetres) between the UAS L quality point cloud and the TLS point cloud

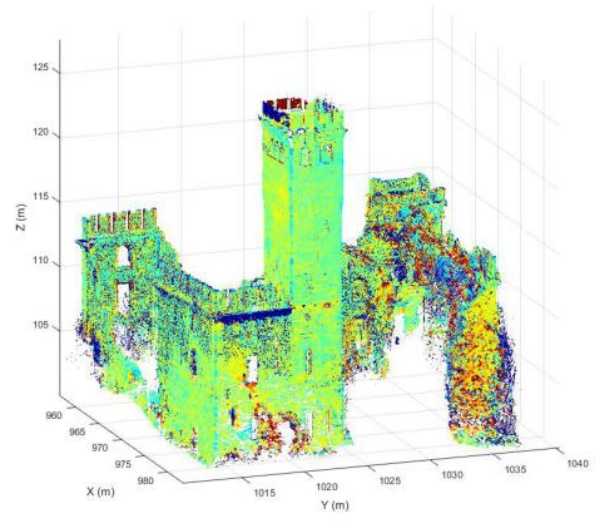

Fig. 12 Differences (in centimetres) between the UAS M quality point cloud and the TLS point clouds

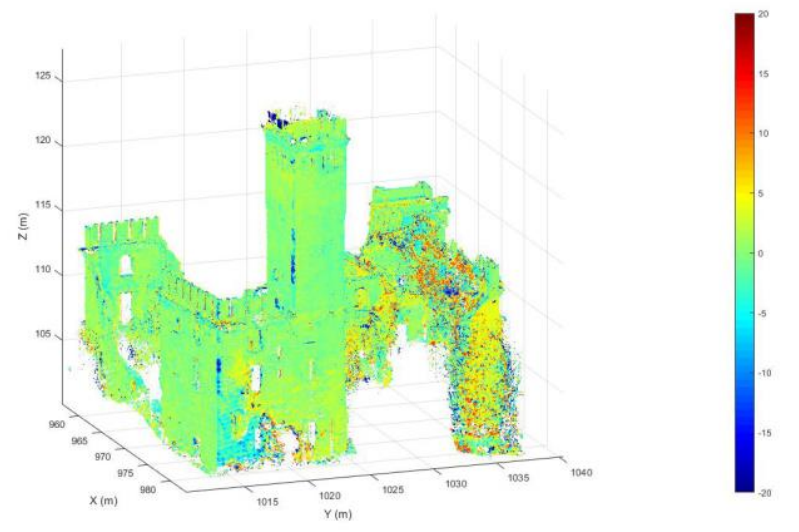

Fig. 13 Differences (in centimetres) between the UAS H quality point cloud and the TLS point clouds

Despite the L dense cloud can be built even on-site in few minutes, and the Gaussian distribution has shown a positive trend, the authors are cautious in asserting its metric effectiveness. In facts, the number of points is very few and the spacing between points is too large to consider this cloud reliable.
An increment of the image quality to generate the dense cloud results in a more detailed description of the investigated object. Starting from M point cloud quality, it is possible to use the generated $3 \mathrm{D}$ data for a preliminary evaluation of the volumes and the dimensions of the structures, with an accuracy in the order of few centimetres. Hence, considering that the time for generating the $M$ points cloud is 1.5 hours, whereas it is about 11 hours for $\mathrm{H}$, a M dense cloud generation is suggested for the overall structure assessment. On the other hand, an H processing is suggested for those portions of the structure whose analyses require a higher amount of details.

In those case for which is difficult to materialize GCPs, the authors recommend (if possible) to enlarge the surveyed area, e.g. up to the UAS pilot ground station, which should be located within a maximum radius of 500 meters from the surveyed object. However, if this is not possible, the presence of an object with known dimensions, e.g. a measurement instrument, in the scene is suggested, to ensure that the model is at least scaled.

\subsection{Techniques for measurements on the 3D model}

As already mentioned, Photogrammetry is an appropriate technique to evaluate also the volume of ruins and collapsed portions. In particular, an important advantage is represented by the possibility of obtaining information of the top of the structures, while this result is not achievable with TLS.

A comparison has been performed to understand the reliability of the different point clouds with respect to the volume computation. In order to perform a homogeneous comparison and due to the lack of the top portions in the TLS point cloud, the lateral surfaces have been used as limits and the volume included between them has been computed.

The sample taken into account in the tests is located on the tower, included between the North and the South façade (Fig. 14).

Fig. 14 Portion taken into account for the volume computation

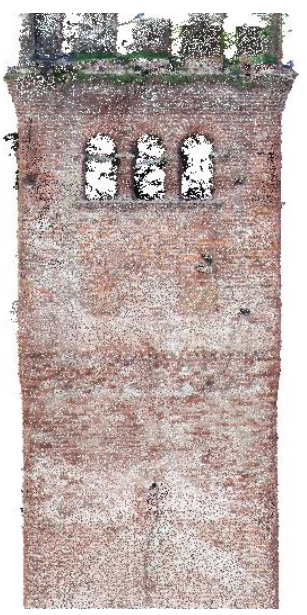

The comparison has been performed by means of the open source software CloudCompare.

For each point cloud, the South and North side of the tower have been used as ground and ceil, respectively, to compute the volume. The option interpolate has been applied to fill empty cells of both the considered surfaces. The grid step has been set equal to the space between points evaluated in 
paragraph 3.3, which corresponds to about $1 \mathrm{~cm}$ for TLS point cloud, $2 \mathrm{~cm}$ for $\mathrm{H}, 4 \mathrm{~cm}$ for $\mathrm{M}$ and $9 \mathrm{~cm}$ for $\mathrm{L}$. The assigned value for each pixel (cell height) has been computed considering the average value.

A volume of about $270 \mathrm{~m}^{3}$ has been computed, with differences located within the range of $(1 \div 5) \%$ between TLS and photogrammetric dense clouds of decreasing quality. The result shows small differences, representative of a reliable behaviour of the analysed clouds.

\section{DATA DISTRIBUTION}

Once the final products are obtained, the issue of data distribution has to be considered. Typically, the end-users are not able to manage the final outputs. Dealing with meshes and point clouds requires several skills and knowledge, including data formats, the use of specific software, how to retrieve derived information from models (e.g. measurements, sections, colour values, etc.) and how to manage data characterized by enormous sizes. In this regard, hardware with certain specifications and performances (e.g. processor, RAM, graphic card, etc.) are necessary to better handle this kind of data.

For these reasons, the outputs delivered to the end-users are usually 2D products, like plans, sections, orthophotos and Digital Elevation Models (DEMs), which are easier to manage with the most commonly used Computer-Aided Drafting (CAD) software and Geographical Information System (GIS) applications. This leads to an inevitable loss of related information given by the nature of the survey itself (e.g. colours, precision, resolution, etc.)

Moreover, in case of emergency, sharing simultaneously the results with all the institutions and operators involved can be very useful to accelerate the data distribution, hence the operations. Web publication of meshes and point clouds allows users to easily navigate, query and analyse $3 \mathrm{D}$ data without requiring any specific skills.

For all these reasons, a Web-based approach has been chosen and tested.

\subsection{Web publication}

Thanks to recent software and hardware advancements, dealing with $3 \mathrm{D}$ products on Web is easier and it permits a widespread and simple distribution of the survey results.

The Web publication of high-resolution models and point clouds has been tested using two different and well-known tools: 3D Heritage Online Presenter (3DHOP) and Potree. The former (http://vcg.isti.cnr.it/3dhop/), developed by the Visual Computing Lab of ISTI-CNR (Pisa, Italy), is a software framework for the creation of interactive Web presentation of high-resolution mesh. The latter (http://potree.org/), developed at the Institute of Computer Graphics and Algorithms of Technische Universität (Vienna, Austria), is a Web-based renderer for large point clouds. Both are free and open source packages, which provide ready-to-use components and functions for the Web visualisation of $3 \mathrm{D}$ data. These characteristics, combined with the possibility of properly customizing the resulting Web page with basic programming knowledge, are the reasons why these two tools have been chosen for testing. 3DHOP (Potenziani et al., 2015) is based on the WebGL subset of HTML5 and on SpiderGL, a Javascript library for advanced computer graphic programming. It is crossplatform and works on the most common browser (e.g. Google Chrome, Mozilla Firefox, Internet Explorer, Safari and Opera) without the need of additional plugins. Also Potree (Schuets, 2016) is based on WebGL, hence it works on the most known browsers without the need to install thirdparty applications.

Both 3DHOP and Potree are based on multi-resolution data structure, which is generally composed by different chunks with different levels of detail. The multi-resolution approach makes the Web visualization faster, but it requires a preprocessing phase for models and point clouds.

Both 3DHOP and Potree provide their own executable to convert single-resolution data into their specific multiresolution file formats, ready to be published on Web. By default, 3DHOP provides many tools for a basic navigation of the model, while other ready-to-use functions (e.g. sections, measure of distance, clickable hotspots, etc.) can be added, as shown in Fig. 15. In this case, basic programming skills are required but the detailed instructions of the available documentation make this step easier even for unexperienced developers. The framework can be used as a simple viewer if any interaction with the Web page is configured, but it can be connected to other active element of the Web page modifying Javascript and CSS/HTML codes.

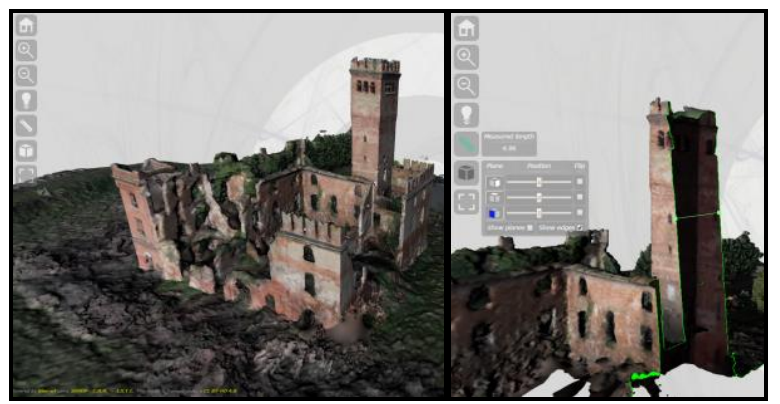

Fig. 15 Mesh of the Castle of Casalbagliano published with 3DHOP: two different points of view and examples of addons tools

About Potree, most of the tools are provided by default resulting in a complex Web viewer. Navigation and measure tools (angles, areas, volumes, distances, etc.), elevation profile and other style functions are available with no configuration, as shown in Fig. 16, while more specific tools (e.g. annotations and overview map) require some changes in the code from the developer. Because of the lack of documentation (only example codes are available), the customization of Potree viewer needs good programming skills.

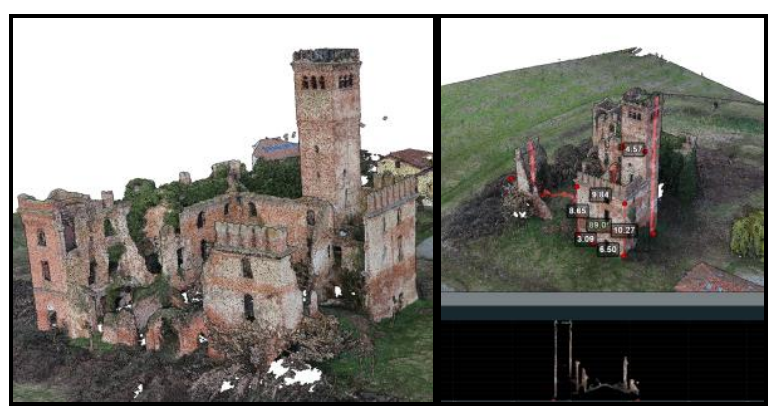

Fig. 16 3D point clouds of the Castle of Casalbagliano published with Potree: a general view (on the left) and the interface for elevation profile (on the right) 
Thanks to their open source nature, both 3DHOP and Potree Web viewers are characterized by high flexibility, which allows creating customized renderer with different levels of complexity, depending on the developer's programming skills.

In conclusion, these two packages provide a useful and complete instrument for data distribution, simplifying the use of meshes and point clouds for all involved operators.

\section{CONCLUSIONS}

This paper outlines a workflow useful for choosing the most suitable operative strategy in case of emergency survey. UAS photogrammetry has proven to be the preferred technique in such scenario, as it allows high accuracy to be achieved and reduced risks for the operators. Moreover, this technique is fast and cheap, allows to inspect the object from above and is characterized by a high efficiency and optimization of resources.

The analysed case study was chosen because of its likeness to an emergency scenario, which is here simulated. In fact, the real risk of collapse makes impossible the approach to the inner part of the Castle.

The impact of different processing parameters has been evaluated (e.g. image acquisition geometry, number and distribution of the GCPs and image quality in the different processing phases) to understand their influence on the final metric accuracy of the delivered 3D models and the impact on the processing time. All the tests have been carried out using Agistoft PhotoScan $\odot$, one of the most commonly used commercial software.

Concerning the requested time for computing the External Orientation (EO) and the dense cloud generation, the number of images in the dataset clearly influences them. In order to optimize the computational times, it is important to evaluate which point of view can guarantee a complete reconstruction of the investigated object, together with the desired GSD.

The number of GCPs is not very influential in terms of processing time for both EO computation and dense cloud generation, but the GCPs distribution needs to be carefully assessed. Reducing the amount of GCPs makes surveying faster and their detection on the photos easier by the operator with comparable final quality. However, a large number of GCPs is helpful in detecting eventual outliers.

In addition, the possibility of GCPs materialization on the ground could be probably compromised by the bad state of the site and the inaccessibility of the area. In this case, some strategies are recommended, for example the extension of the survey area or the scaling by means of an object with known dimensions.

The processing quality (i.e. image downscaling) should be decided taking into account the expected final accuracy, as well as the operative conditions. In particular, in the alignment phase the choice of $\mathrm{M}$ or $\mathrm{H}$ depends not only on the speed of calculation, but also on the complexity of the object and its texture, in order to obtain a correct matching. In the dense cloud generation, it is recommended to use medium quality when the aim is the reconstruction of the global shape of the investigated structure, while high quality can be used for those areas where a high level of detail is required.

In light of what emerged from the analysis carried out, the suggested workflow of the survey operations in an emergency scenario typically should include: (1) the acquisition of a restrained image dataset from the optimal point of view; (2) the processing of the alignment phase in medium image quality, to speed up the computation of about $30 \%$; (3) the reconstruction of the dense cloud with the use of the medium image quality for the overall structure assessment and the high image quality only for detailed portions.

Finally, some techniques for Web publication of 3D models have been tested. The 3DHOP and Potree tools, available as open source software, allow the navigation and the measurement of both meshes and point clouds respectively. The availability of these platforms could help operators to better enhance their work, and disseminate it to the endusers, who could easily use the published data.

\section{ACKNOWLEDGEMENTS}

This work comes from the master thesis of one of the authors. The authors would like to thank for the provided support:

- Comune di Alessandria, in particular Arch. Marco Genovese and Geom. Gianfranco Ferraris, for their availability;

- Soprintendenza belle arti e paesaggio della Provincia di Alessandria, in particular Arch. Luigi Pedrini and Dott. Valentina Uras;

- the co-supervisors of the thesis: Eng. Serena Cattari and Arch. Rita Vecchiattini, respectively at DICCA and DAD Departments at the Genoa University.

\section{REFERENCES}

3DHOP, 2018, http://vcg.isti.cnr.it/3dhop (access on $15^{\text {th }}$ January 2018), see for instance http://www.gter.it/3dhosting/casalbagliano/

Agisoft PhotoScan $\odot$, 2018, http://www.agisoft.com/ (access on $15^{\text {th }}$ January 2018)

Achille, C., Adami, A., Chiarini, S., Cremonesi, S., Fassi, F., Fregonese, L., Taffurelli, L., 2015. UAV-based photogrammetry and integrated technologies for architectural applications-Methodological strategies for the after-quake survey of vertical structures in Mantua (Italy). Sensors 15(7), pp. 15520-15539.

Ballarin, M., Buttolo, V., Guerra, F., Vernier, P., 2013. Integrated surveying techniques for sensitive areas: San Felice sul Panaro. ISPRS Annals of the Photogrammetry, Remote Sensing and Spatial Information Sciences 5, W1.

Ballarin, M., Balletti, C., Faccio, P., Guerra, F., Saetta, A., Vernier, P., 2017. Survey methods for seismic vulnerability assessment of historical masonry buildings. Int. Arch. Photogramm. Remote Sens. Spatial Inf. Sci., XLII-5/W1, 5559, https://doi.org/10.5194/isprs-archives-XLII-5-W1-552017

Bruno, F., Bruno, S., De Sensi, G., Luchi, M. L., Mancuso, S., Muzzupappa, M., 2010. From 3D reconstruction to virtual reality: A complete methodology for digital archaeological exhibition. Journal of Cultural Heritage 11(1), pp. 42-49.

Barone, Z. Nuccio, G., 2017. For a conscious fruition of the Cultural Heritage of ancient Noto (Sicily). EFIAN project as opportunity for valorisation. Int. Arch. Photogramm. Remote Sens. Spatial Inf. Sci., XLII-5/W1, 89-97, https://doi.org/10.5194/isprs-archives-XLII-5-W1-89-2017 
Brutto, M. L., Garraffa, A., Meli, P., 2014. UAV platforms for cultural heritage survey: first results. ISPRS Annals of the Photogrammetry, Remote Sensing and Spatial Information Sciences 2(5), p. 227.

Chiabrando, F., Di Lolli, A., Patrucco, G., Spanò, A., Sammartano, G., Teppati Losè, L., 2017. Multitemporal 3D modelling for Cultural Heritage emergency during seismic events: damage assessment of S. Agostino church in Amatrice (RI). Int. Arch. Photogramm. Remote Sens. Spatial Inf. Sci., XLII-5/W1, 69-76, https://doi.org/10.5194/isprsarchives-XLII-5-W1-69-2017

CloudCompare, 2018, http://cloudcompare.org/ (access on $15^{\text {th }}$ January 2018)

Fagandini, R., Federici, B., Ferrando, I., Gagliolo, S., Pagliari, D., Passoni D., Pinto, L., Rossi, L., Sguerso, D., 2017. Evaluation of the Laser Response of Leica Nova Multistation MS60 for 3D Modelling and Structural Monitoring. In International Conference on Computational Science and Its Applications, pp. 93-104

Fregonese, L., Campera, A., Scala, B., Adami, A., 2017. The 2012 earthquake: an abacus of surveys and interventions in Mantua churches. Int. Arch. Photogramm. Remote Sens. Spatial Inf. Sci., XLII-5/W1, 31-38, https://doi.org/10.5194/isprs-archives-XLII-5-W1-31-2017

Gagliolo, S., Fagandini, R., Federici, B., Ferrando, I., Passoni, D., Pagliari, D., Pinto, L., Sguerso, D., 2017. Use of UAS for the conservation of historical buildings in case of emergencies. International Archives of the Photogrammetry, Remote Sensing and Spatial Information Sciences, Vol. XLII$5 / W 1$, p. $81-88$.

Grün, A., Remondino, F., Zhang, L., 2004. Photogrammetric reconstruction of the great Buddha of Bamiyan, Afghanistan. The Photogrammetric Record 19.107, pp. 177199.

Lague, D., Brodu, N., Leroux, J., 2013. Accurate 3D comparison of complex topography with terrestrial laser scanner: application to the Rangitikei canyon (N-Z). ISPRS Journal of Photogrammetry and Remote Sensing 82, pp. 1026

Meyer, D., Hess, M., Lo, E., Wittich, C. E., Hutchinson, T. C., Kuester, F., 2015. UAV-based post disaster assessment of cultural heritage sites following the 2014 South Napa Earthquake. Digital Heritage, Vol. 2, pp. 421-424.

Oreni, D., Brumana, R., Della Torre, S., Banfi, F., 2017. Survey, HBIM and conservation plan of a monumental building damaged by earthquake. Int. Arch. Photogramm. Remote Sens. Spatial Inf. Sci., XLII-5/W1, 337-342, https://doi.org/10.5194/isprs-archives-XLII-5-W1-337-2017

Potree, 2018, http://potree.org/ (access on 15 $5^{\text {th }}$ January 2018), see for instance http://www.gter.it/3dhosting/casalbagliano/index_pot.html

Potenziani, M., Callieri, M., Dellepiane, M., Corsini, M., Ponchio, F., Scopigno, R., 2015. 3DHOP: 3D Heritage Online Presenter. Computers \& Graphics, Volume 52, pp. 129-141, ISSN 0097-8493.
Radicioni, F., Matracchi, P., Brigante, R., Brozzi, A., Cecconi, M., Stoppini, A., Tosi, G., 2017. The Tempio della Consolazione in Todi: integrated geomatic techniques for a monument description including structural damage evolution in time. Int. Arch. Photogramm. Remote Sens. Spatial Inf. Sci., XLII-5/W1, 433-440, https://doi.org/10.5194/isprsarchives-XLII-5-W1-433-2017

Remondino, F., 2011. Heritage recording and 3D modeling with photogrammetry and 3D scanning. Remote Sensing 3(6), pp. 1104-1138.

Sauerbier, M., Eisenbeiss, H., 2010. UAVs for the documentation of archaeological excavations. International Archives of Photogrammetry, Remote Sensing and Spatial Information Sciences 38 (Part 5), pp. 526-531.

Schuetz, M., 2016. Potree: Rendering large point clouds in Web browser.

Themistocleous, K., Ioannides, M., Agapiou, A., Hadjimitsis, D. G., 2015. The methodology of documenting cultural heritage sites using photogrammetry, UAV, and 3D printing techniques: the case study of Asinou Church in Cyprus. In Third International Conference on Remote Sensing and Geoinformation of the Environment, pp. 953510-953510.

Wojciechowski, R., Walczak, K., White, M., Cellary, W., 2004. Building virtual and augmented reality museum exhibitions. Proceedings of the ninth international conference on 3D Web technology, ACM.

Xu, Z., Yang, J., Peng, C., Wu, Y., Jiang, X., Li, R., Zeng, Y., Gao, Y., Liu, S., Tian, B., 2014. Development of an UAS for post-earthquake disaster surveying and its application in Ms7. 0 Lushan Earthquake, Sichuan, China. Computers \& Geosciences 68, pp. 22-30.

Yastikli, N., 2007. Documentation of cultural heritage using digital photogrammetry and laser scanning. Journal of Cultural Heritage 8.4, pp. 423-427.

Yilmaz, H. M., Yakar, M., Gulec, S. A., Dulgerler, O. N., 2007. Importance of digital close-range photogrammetry in documentation of cultural heritage. Journal of Cultural Heritage 8.4, pp. 428-433. 